\title{
Editorial: Special issue on dependable peer-to-peer systems
}

\author{
Lu Liu • Jie Xu
}

Received: 11 December 2009 / Accepted: 23 December 2009 / Published online: 26 January 2010

(C) Springer Science+Business Media, LLC 2010

Keywords Dependability $\cdot$ Peer-to-peer systems

As an emerging technology, peer-to-peer (P2P) systems attract attention worldwide, ranging from casual Internet users to venture capitalists. At the same time, innovations of P2P systems also offer many interesting avenues of research for scientific communities. As a major design pattern for future systems opposite to the traditional clientserver paradigm, research on P2P networks is extremely important and could significantly change the way of use of computer networks.

However, P2P is sometimes regarded as a destructive technology rather than a constructive technology by Internet Service Providers (ISPs). "How to harness the power of P2P?" and "how to design dependable P2P systems for sustainable provision of services and integration of services?" remain fundamental problems. There are strong needs for improving the dependability of P2P systems to make them more secure and robust for service

\section{Liu $(\square)$}

Department of Computer Communications,

School of Engineering and Information Sciences,

Middlesex University,

London NW4 4BT, UK

e-mail: 1.liu@mdx.ac.uk

L. Liu $\cdot$ J. Xu

School of Computing, Faculty of Engineering,

University of Leeds,

Leeds LS2 9JT, UK sharing and delivery. However, developing dependable systems is difficult because various types of properties can influence the dependability of system. Achieving dependability in a P2P environment is even more complicated due to the lack of centralized servers to monitor and control systems.

The purpose of this special issue is to collate efforts and main achievements that contribute to scalability, availability, reliability, security and maintainability of P2P systems. This special issue focuses on fundamental issues in modelling, simulation and design of dependable P2P systems. This special issue contains six high quality papers which were selected from 17 submissions after a peer review process.

Load balancing is a well-known crucial problem in P2P systems. Vinod et al. present a mechanism to optimise server-node ratio for load balancing in P2P networks. This mechanism builds upon a load-balancing scheme, called Enhanced-Reconfigurable-Gnutella-Overlay (ERGO). In this paper, the influence of various parameters such as network load, node capacity, server capacity and the corresponding effects of server-node ratio on metrics such as success of queries, balanced nodes and the cost of achieving the balance have been systematically investigated and discussed. An optimum server-node ratio is ascertained, optimum implying the maximum balance at minimum cost and high success of queries.

Previous measurement studies have shown that some nodes observed in existing P2P networks tend to download a large number of files, but share few files or none at all. 
Sasabe et al. investigate how these selfish nodes' behaviours affect the performance of P2P systems. The authors propose a bargaining approach which has been evaluated through simulations and discuss relationship between selfish node behaviour and system dynamics by using evolutionary game theory. The evaluation results show that the storage capacity model makes a file-sharing system robust to file disappearance independently of the network structures even if nodes behave selfishly. Addressing the similar issues of free-riding and fairness in BitTorrent networks, W. Liu et al. present a Tit-for-Tat model based on game theory. The existing problems of incentive mechanisms in BitTorrent networks have been analysed and an improved method based on Tit-for-Tat strategy has been proposed in this paper. The authors verify the effectiveness of the enhanced Tit-for-Tat strategy in P2P environments through simulations.

Security is essential, but particularly challenging in $\mathrm{P} 2 \mathrm{P}$ systems. Sun et al. investigate the possibilities of creating the DDoS attacks using the different membership management algorithms in P2P systems. The authors evaluate the potential of mitigation schemes in enhancing the resilience of P2P systems through experiments on the Planetlab and also reveal the weakness in the schemes and regimes where they may not be sufficient.

Redundancy is a fundamental technique to improve reliability of P2P systems. Duminuco \& Biersack present an erasure coding scheme called Hierarchical Codes which provide a flexible method of adding redundancy in distributed P2P storage systems. Hierarchical Codes achieve reduced repair traffic offered by replication and higher resilience against failures offered by coding. The simulation results show that Hierarchical Codes require a higher number of repairs but result in a lower amount of repair traffic to be transferred.

Self-organisation could be a good way to solve the control issues in the decentralised P2P architecture. Doulkeridis et al. propose a data-centric self-organizing P2P algorithm which is capable to dynamically identify data clusters on different peer nodes and allocate peer nodes sharing similar shared content to the same super-peer. This improves the overall search performance and increases the dependability of the P2P system.

Finally, we would like to express my sincere thanks to Dr Zongyang Luo, Mr Shengdong Zhang and Mr David Webster for their continuous support and contributions, and to the colleagues who have served as reviewers for the submissions. We also would like to thank Prof Sherman Shen, Dr Heather Yu, Valerie Schofield and Christina Chua for their great guidance and consistent help in the whole process of preparing this special issue.

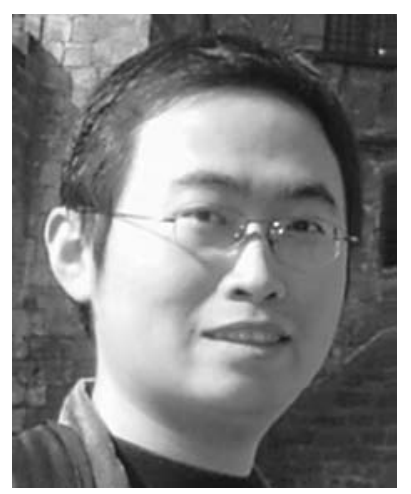

Dr Lu Liu is Lecturer in School of Engineering and Information Sciences at Middlesex University (UK). Before joining Middlesex University, he was Research Fellow in the School of Computing at the University of Leeds (UK), working on NECTISE Project which was an UK EPSRC/BAE Systems funded research project involving ten UK Universities and CoLab Project which was funded by UK EPSRC and China 863 Program. He received a Ph.D degree (funded by UK DIF DTC) from the University of Surrey (UK) and M.Sc. degree from Brunel University (UK). His research interests are in areas of peer-to-peer computing, software engineering and service-oriented computing. Dr Liu has over 30 scientific publications in reputable journals, academic books and international conferences. He won the Best Paper Award at the Realising Network Enabled Capability Conference in 2008. He is on the Editorial Review Board of International Journal of Distributed Systems and Technologies. He served as Co-chair, TPC Member, Advisory Committee Member and Session Chair of many international conferences and workshops. Contact him at Department of Computer Communications, School of Engineering and Information Sciences, Middlesex University, London, NW4 4BT, United Kingdom; 1.liu@mdx.ac.uk

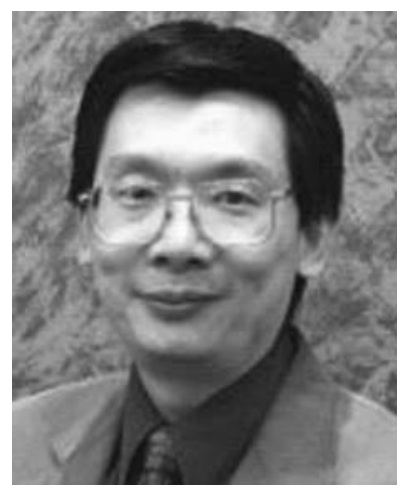

Professor Jie Xu is Chair of Computing at the University of Leeds (UK), Director of Institute for Computational and Systems Science, University of Leeds, and Director of the EPSRC WRG e-Science Centre involving the three White Rose Universities of Leeds, York and Sheffield. He is also a visiting professor at the School of Computing Science, the University of Newcastle upon Tyne (UK) and a Changjiang Scholar visiting professor in Chongqing University (China). He has worked in the field of Distributed Computer Systems for over twenty years and had industrial experience in building large-scale networked systems. Professor $\mathrm{Xu}$ now leads a collaborative research team at Leeds studying Grid and Internet technologies with a focus on complex system engineering, system security and dependability, and evolving system architectures. He is the recipient of the BCS/IEE Brendan Murphy Prize 2001 for the best work in the area of distributed systems and networks. He has led or co-led many key research projects served as Program Chair/PC member of, many international computer conferences. Professor $\mathrm{Xu}$ has published more than 200 edited books, book chapters and academic papers, and has been Editor of IEEE Distributed Systems since 2000. He was Professor of Distributed Systems in Durham University (UK) before he joined the University of Leeds. Contact him at School of Computing, Faculty of Engineering, University of Leeds, Leeds, LS2 9JT, United Kingdom; j.xu@leeds.ac.uk 